\title{
New Electron and Ion Beam Chemistry for Nanotechnology
}

\author{
J.J.L. Mulders* \\ * FEI Electron Optics, Achtseweg Noord 5, 5600 KA Eindhoven, The Netherlands
}

A focused ion beam system or a scanning electron microscope can be equipped with a facility for local, direct delivery of a gas or vapor, close to the sample. With a suitable gas its decomposition, induced by the sample-beam interaction phenomena, results in deposition of one or more of its components. In this way it is possible create a very local deposition of materials such as $\mathrm{Pt}, \mathrm{W}$ or even silicon (di) oxide. This direct deposition method applies to both the ion beam and the electron beam. In many cases researchers try to obtain a very local property such as conductivity, mechanical strength, optical behavior, mass or a chemical or biological activity and usually the expected property is derived from the (known) properties of the bulk material. The depositions however, induced by the electron or ion beam do not have the high, bulk-related purity and hence their property aimed for may be different. By developing new chemistries and additional processes however, the nano scale depositions can become much better in this respect and at the same time the material types can be increased, so the application space gets expanded to, for example, magnetic materials. Traditionally many processes and materials are applied in the semi-conductor industry for circuit-edit applications. For this reason the focus has been on conductivity of materials and therefore on CMOS compatible metals such as W. In nano technology however, the request for nano scale structures is dealing for example with Carbon Nano Tube ohmic contacts, magnetic sensors (AFM tips), small dots as seed centers, and gold structures for biological applications (binding sites for thiol groups). These new applications require the development of new chemistry for the instrument that is practically useable, affordable and safe for men and instrument.

The GIS system

The system that supplies the gas molecules on an FEI based FIB, SEM or DualBeam is a gas injector system (GIS). This is a very compact system that holds the solid or liquid chemical (precursor) in a dedicated, temperature controlled, closed reservoir. The vapor pressure above the precursor equals the pressure that corresponds to the saturated vapor pressure at the adjusted temperature for the specific chemical. In general this relation follows a Clausius-Clapeyron equation: $\log ($ pressure $)=\mathrm{a} / \mathrm{T}$ $+\mathrm{b}$. In many cases however, $\mathrm{a}$ and $\mathrm{b}$ are not known, and a best guess has to be made, using the melting and boiling point instead. The GIS exhaust is formed by a pneumatic safety valve followed by a long needle, and the whole assembly can be inserted very close to the sample (typically 100 - $200 \mathrm{um}$ ), with micron scale repeatability. As the chemical reservoir is actually within the microscope chamber, the system is a mini chemical chamber within the vacuum chamber and therefore very safe. Typically the pressure within the GIS is around $0.1-1 \mathrm{mBar}$ and for higher vapor pressures such as for TEOS (tetraethylorthoslilcate) an extra orifice is used to lower the actual molecular flux and bring it within the typical range of $10^{16}-10^{18}$ molecules $/ \mathrm{cm}^{2}$.s. In daily use the system is operated with three commands only and the resulting flux is very constant, whereas the lateral density profile is very broad (100's of microns) resulting in very good homogeneity of the depositions on the sample, over the area of interest. The one-to-one relation for the chemical and crucible (injector) guarantees a system that is absolutely free from any cross-contamination. Any new chemical for the GIS, only has to have the proper vapor pressure range and chemical compatibility with the crucible and it should be safe for the instrument and its environment. As an example, W deposition can also be realized using $\mathrm{WF}_{6}$ in stead of $\mathrm{W}(\mathrm{CO})_{6}$ : the absence of carbon in the precursor 
molecule and the presence of fluorine result in a high purity for $\mathrm{W}$, but the chamber microscope and all its internal (stage) parts will be subject to a higher level of corrosion.

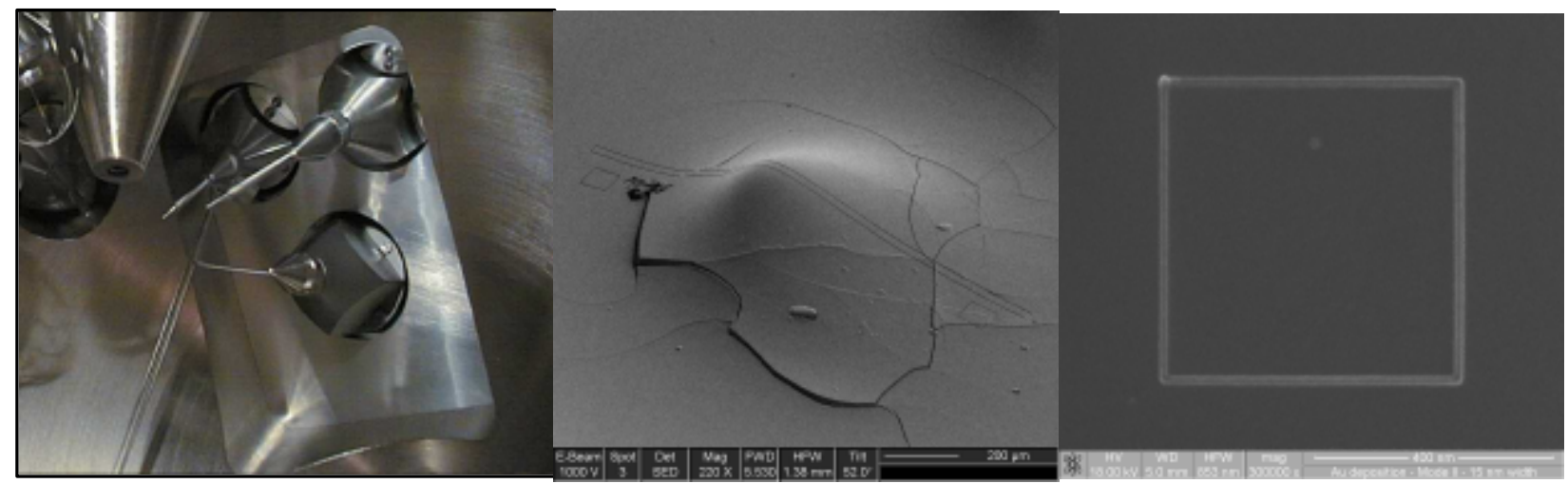

Figure 1a) Chamber view of 3 GIS systems showing the crucible and needle (retracted position). Figure 1b) The precursor density distribution over the surface of the sample, measured by cryofreezing of the precursor flux and milling a double trace over the full area of interest (horizontal field width $=1.2 \mathrm{~mm}$, GIS insertion position top left corner). Figure 1c) Gold deposition of a $500 \mathrm{~nm}$ frame with a 15 x $15 \mathrm{~nm}$ line size.

Development aspects of a new process

Selection of a new precursor quickly focuses on extension of the metal-carbonyl compounds equivalent to for example $\mathrm{W}(\mathrm{CO})_{6}$. So a need for Nickel or Iron deposition would suggest the use of the compounds $\mathrm{Fe}(\mathrm{CO})_{5}$ and $\mathrm{Ni}(\mathrm{CO})_{4}$. These compounds however, are very toxic and need special attention and even special transport. The easy release of the carbon monoxide upon handling makes these chemicals unsuited for direct application without extensive additional protection. Therefore less toxic components are very much preferred.

The standard chemical for Pt deposition (MeCpPtMe3) also produces carbon in the deposit and therefore the tetrakis $\mathrm{Pt}$ may be an alternative option as it contains no carbon but $\mathrm{PF}_{3}$ groups in stead. It is expected that the use of this precursor results in a higher-purity Pt deposition. However, it will be necessary to prove the long-term stability of the instrument for the phosphine groups. And as the vapor pressure for tetrakis Pt is quite high, the material is more hazardous and the chemical is sensitive to air it is not so straightforward to use it in a daily routine. So switching from one chemical to another may seem simple, but many aspects have to be taken into account. An example of a relatively harmless gold precursor is shown in Figure 1c. This material has been applied in the GIS and the process, stability and usefulness for creation of very small nano scale gold structures has been proven.

\section{Conclusion}

The need for new beam chemistry for nano scale deposition is growing. Within the boundaries of the Gas Injection System hardware and the required safety aspects it is possible to introduce several new precursor materials to obtain a new category of materials. Constant development in this area will bring new capabilities for researchers in the field of nano technology. 